\title{
Misconception and Misbehaviour towards Helicobacter pylori is Leading to Major Spread of Illness
}

\author{
Abdullah M Nasrat ${ }^{1^{*}}$, Salwa AM Nasrat ${ }^{2}$, Randa M Nasrat ${ }^{3}$ and Mohammad M Nasrat ${ }^{3}$ \\ ${ }^{1}$ Department of Surgery, Balghsoon Clinic, Jeddah, KSA \\ ${ }^{2}$ Department of Physical Therapy, Cardiac Surgery Academy, Cairo, Egypt \\ ${ }^{3}$ Department of Internal Medicine, Helwan General Hospital, Helwan, Egypt
}

*Corresponding Author: Abdullah M Nasrat, Department of Surgery, Balghsoon Clinic, PO Box 5261, Jeddah 21573, KSA, Tel: + 966 (012) 667 3645; E-mail: abdullahnasrat@hotmail.com

Rec date: July 27, 2015 Acc date: August 18, 2015 Pub date: September 24, 2015

Copyright: (C) 2015 Nasrat AM, et al. This is an open-access article distributed under the terms of the Creative Commons Attribution License, which permits unrestricted use, distribution, and reproduction in any medium, provided the original author and source are credited.

\begin{abstract}
This study aimed to demonstrate that the antibiotic violence has rendered a domestic bug (Helicobacter pylori) to become wild in attitude instead of getting rid of it.

The widespread prevalence and the challenges constituted by $H$. pylori; namely its close relation to acid peptic disease, gastric carcinoma and lymphoma have led to the widely-established medical concept that $H$. pylori eradication should be a necessary attempt. The flare up of these medical problems indicates that the current combined antibiotic therapy is not an effective measure to control all the challenges related to the stomach bug. $H$. pylori colonized the stomach since an immemorial time; the antibiotics could force $H$. pylori to migrate to another shelter which would render it to become a source of illness.
\end{abstract}

The scientific interest of this study was focused on three groups of clinical conditions associated with $H$. pylori dyspepsia; chronic and recurrent colitis, uncontrolled hypertension under medication and newly discovered diabetes mellitus in adults. Eradication of $H$. pylori was done by natural measures; colon care and colon clear.

All patients became free of any dyspeptic symptoms. The integral colonic function has been easily resumed in 33 patients. 15 patients were able to quit their antihypertensive pills and maintain normal blood pressure values. The diabetic condition has been successfully and permanently corrected in 10 newly discovered patients.

On conclusion, Migration of $H$. pylori is associated with reasons of etiologic pathology due to accumulation of toxic amounts of ammonia somewhere in the body.

Keywords: Helicobacter pylori; Antibiotic violence

\section{Introduction}

The widespread prevalence and the challenges constituted by Helicobacter pylori; namely its close relation to acid peptic disease, gastric carcinoma and lymphoma have led to the widely-established medical concept that $H$. pylori eradication should be a necessary attempt. The annual cost associated with peptic ulcer disease in the United States is estimated to be 6 billion dollars and gastric cancer kills over 700,000 people every year in the world $[1,2]$.

H. pylori is an extremely common bacterium which is considered by quite many investigators upon statistical basis or relative association with some diseases to be pathogenic bacterium that is able to alter the host physiology; subverting its immune response and allowing it to persist for the life of the host. The prevalence of $H$. pylori remains high; over $50 \%$ in most of the world, although existence rates are dropping in some developed countries. The drop in $H$. pylori prevalence could be a double-sided matter; reducing the incidence of gastric diseases while increasing the risk of allergies, auto-immunity and esophageal diseases $[2,3]$.
The latest reports in literature demonstrate a definite flare up of many medical challenges related to $H$. pylori through immune or different unknown reasons. Thyroiditis, autoimmune pancreatitis, immune thrombocytopenic purpura and acute inflammatory demyelinating polyradiculoneuropathy are examples of these challenges [3-6]. These autoimmune medical challenges related to $H$. pylori are sufficient to render the matter that $H$. pylori can reside hidden somewhere in the body be taken seriously.

The list of diseases potentially related to $H$. pylori continues to grow, however, explanations of how $H$. pylori could contribute to extragastric diseases lag far behind clinical studies. A number of host factors and $H$. pylori virulence factors act together to determine which individuals are most predisposed and susceptible or are at the highest risk of disease. These factors include bacterial cytotoxins and polymorphisms in host genes responsible for directing the immune response $[2,7,8]$.

H. pylori could migrate or get forced or migrate to the colon under the influence of antibiotic violence where it will continue producing ammonia for a reason or no reason, unopposed or buffered by any acidity unlike in the stomach leading to accumulation of profuse amounts of ammonia; gastric juxta-mucosal ammonia of $H$. pylori is 
strictly liberated in response to any acidity approaching the gastric mucosa. Accumulation of excess ammonia in the body is known toxic that could lead to adverse biological effects in the body among predisposed disadvantaged population. Ammonia is smooth muscle tonic and therefore; excess ammonia in the colon could be spastic causing multiple colonic spasms [9-12].

\section{Aim}

This study aimed to demonstrate that the antibiotic violence has rendered a domestic bug ( $H$. pylori) to become wild in attitude instead of getting rid of it.

\section{Design and Setting}

Prospective study done in Balghsoon Clinics in Jeddah/Saudi Arabia during 2012/2013

\section{Patients and Methods}

The scientific interest of this study was focused on three groups of clinical conditions associated with $H$. pylori dyspepsia; chronic and recurrent colitis, uncontrolled hypertension under medication and newly discovered diabetes mellitus in adults. The patients were randomly included in the study without selection. The rectal spasm was detected by proctoscopy or sigmoidoscopy, while the multiple colonic spasms were demonstrated by colonoscopy. The management of cases constituted eradication of $H$. pylori by natural measures employing the senna purge and vinegar therapy.

\section{Results}

All patients became free of any dyspeptic symptoms. The integral colonic function has been easily rectified in 33 patients. 15 hypertensive patients were able to quit their antihypertensive pills and maintain normal blood pressure values although they were inadequately controlled in spite of regular follow up of medications and extreme carefulness about their life style. The diabetic condition has been successfully and permanently corrected in 10 newly discovered cases.

\section{Ethical Considerations}

An informed signed consent was taken from all patients, they were made aware about safety of the natural colon clear remedy; they were free to quit the study whenever they like. The research proposal was approved and the study followed the rules of the Research Ethics Committee of Balghsoon Clinics in Jeddah, Saudi Arabia.

\section{Discussion}

Most of the diabetic and hypertensive patients in the world are inadequately controlled in spite of regular follow up of medications and strict carefulness about their style of life; this could further indicate the presence of a missed underlying pathology [9-11].

A general impression has developed that the antibiotic violence has forced $H$. pylori to migrate in panic to the colon and the rectum. Migration of $H$. pylori will be accompanied by accumulation of profuse toxic amounts of ammonia in the colon which will lead to multiple colonic and a high rectal spasm. This matter is manifested by marked constipation and passage of small pieces of dried stool which have been observed as integral intensity of $H$. pylori-related dyspepsia. These spasms are so resistant to ordinary laxative measures leading to loss of the integral colonic function and set up of a state of irritable or spastic colitis [9].

The development of multiple colonic spasms with subsequent severe constipation and interference with the integral colonic function will establish a colonic re-absorption error with retention of fluids, salts and toxins inside the body. This could lead to hypertension which is expected to remain inadequately controlled without correction of the underlying colonic re-absorptive error. Hypertension, a disease of rich, is now flaring up as a challenge among poor population. Some reports consider hypertension in developing countries a consequence of progress and life style changes. In spite of that, traditional risk factors do not appear fully sufficient to explain the rising figures of hypertensive illness $[11,13,14]$.

Accumulation of profuse toxic amounts of ammonia in the colon constitutes a biological toxic stress to the body that could lead to stress diabetes among disadvantaged susceptible people. Administration of oral hypoglycemic drugs to a stressed pancreas means an insistence to flog a tired horse turning a potential condition into an established chronic illness with consequent flare up of the diabetic phenomena all over the world. Traditional risk rules are not sufficient to explain the dramatic spread of diabetes all over the world; this indicates that traditional measures employed to control the problem can never be adequate or decisive $[9,10]$.

Misconception of most investigators about the nature of $H$. pylori and their misbehavior towards it is possibly leading in great part to the major spread of pathologic conditions associated with $H$. pylori existence. $H$. pylori colonized the stomach since an immemorial time; [7] as if both the stomach and the bug used to live together in peace, harmless to each other. This also indicates that the commonest natural habitat of $H$. pylori is the stomach; if $H$. pylori is kicked outside the stomach by antibiotics, it will become a foreign structure the tissues and is rendered a poison itself by causing local tissue pathology or becomes a source of poison by encoding autoimmunity or by leading to accumulation of profuse toxic amounts of ammonia somewhere in the body [7,9]. Recurrence of $H$. pylori existence is un-avoidable or hardly avoidable; $[7,8]$ as if it is a matter of natural existence of a bacterium. Existence of abnormal-behavior $H$. pylori strains is essentially a sanitary conflict before it is a medical challenge, [7] sanitary problems are treated with antiseptic measures but not antibiotics. If investigators and therapists keep these findings in consideration, most of the challenges associated with $H$. pylori would disappear. Moreover, the continued flare up of the medical problems associated with $H$. pylori indicates that the current combined antibiotic therapy is not an effective measure to control all the challenges related to the stomach bacterium.

The senna leaves extract purge effectively and readily kills and expels all migrated colonic $H$. pylori strains. Vinegar therapy maintains colon clear by interference with re-set up of abnormal-behavior $H$. pylori strains due to interference with the energy metabolism and respiratory chain metabolism of $H$. pylori via inhibition of the pyruvate dehydrogenase complex according to the rules of feedback regulation and product inhibition with consequent inhibition of pyruvate metabolism which is the main source of energy production by for $H$. pylori [7,9-12].

The dramatic response achieved in this study confirms the concept that the etiologic pathology associated with $H$. pylori was simply the 
Citation: Nasrat AM, Nasrat SAM, Nasrat RM, Nasrat MM (2015) Misconception and Misbehaviour towards Helicobacter pylori is Leading to Major Spread of Illness. Gen Med (Los Angel) S1: S1-002. doi:10.4172/2327-5146.1000S1-002

Page 3 of 3

colon-spastic and toxic effect of ammonia produced by H. pylori in the colon. The integral colonic function was resumed by rectifying the multiple colonic spasms, blood pressure values were maintained with quit of antihypertensive medications by correction of the colonic reabsorptive error and recovery of the newly discovered diabetic condition was achieved by elimination of the biological toxic stress situation via mere natural eradication of $H$. pylori from the colon.

\section{Conclusion}

It seems that most of the budget of searching and researching after H. pylori should be redirected towards raising the standard of life and ensuring sanitary water supply for poor population, patient and family education as regards misbehaviour in food habits and antibiotic abuse, and orientation in primary health care units as concerns natural measures towards $H$. pylori dyspepsia.

\section{References}

1. Ge Z (2002) Potential of fumarate reductase as a novel therapeutic target in Helicobacter pylori infection. Expert Opin Ther Targets 6: 135-146.

2. Testerman TL, Morris J (2014) Beyond the stomach: an updated view of Helicobacter pylori pathogenesis, diagnosis, and treatment. World J Gastroenterol 20: 12781-12808.

3. Segni M, Borrelli O, Pucarelli I, Delle Fave G, Pasquino AM, et al. (2004) Early manifestations of gastric autoimmunity in patients with juvenile autoimmune thyroid diseases. J Clin Endocrinol Metab 89: 4944-4948.

4. Kountouras J, Zavos C, Chatzopoulos D (2005) A concept on the role of Helicobacter pylori infection in autoimmune pancreatitis. J Cell Mol Med 9: 196-207.
5. Kountouras J, Deretzi G, Zavos C (2005) Association between Helicobacter pylori infection and acute inflammatory demyelinating polyradiculoneuropathy. Eur J Neurol 12: 139-143.

6. Veneri D, Krampera M, Franchini M (2005) High prevalence of sustained remission of idiopathic thrombocytopenic purpura after Helicobacter pylori eradication: a long-term follow-up study. Platelets 16: 117-119.

7. Farinha P, Gascoyne RD (2005) Helicobacter pylori and MALT lymphoma. Gastroenterology 128: 1579-1605.

8. Cirak MY, Ozdek A, Yilmaz D, Bayiz U, Samim E, et al. (2003) Detection of Helicobacter pylori and its CagA gene in tonsil and adenoid tissues by PCR. Arch Otolaryngol Head Neck Surg 129: 1225-1229.

9. Nasrat AM (2009) The world misconception and misbehavior towards Helicobacter pylori is leading to major spread of illness. The 7th AntiAging Medicine World Congress, Monte-Carlo, Monaco.

10. Nasrat SAM, Nasrat RM, Nasrat MM (2015) The dramatic spread of diabetes mellitus worldwide and influence of Helicobacter pylori. General Med J 3: 159-162.

11. Nasrat SAM, Nasrat AM (2015) An alternative approach for the rising challenge of hypertensive illness via Helicobacter pylori eradication. J Cardiol Res 6: 221-225.

12. Nsarat RM, Nasrat MM, Nasrat AM (2015) Improvement of idiopatic cardiomyopathy after colon clear. J Cardiol Res 6: 249-254.

13. Bakris G, Hill M, Mancia G, Steyn K, Black HR, et al. (2008) Achieving blood pressure goals globally: five core actions for health-care professionals. A worldwide call to action. J Hum Hypertens 22: 63-70.

14. Reddy KS, Naik N, Prabhakaran D (2006) Hypertension in the developing world: a consequence of progress. Curr Cardiol Rep 8: 399-404. 